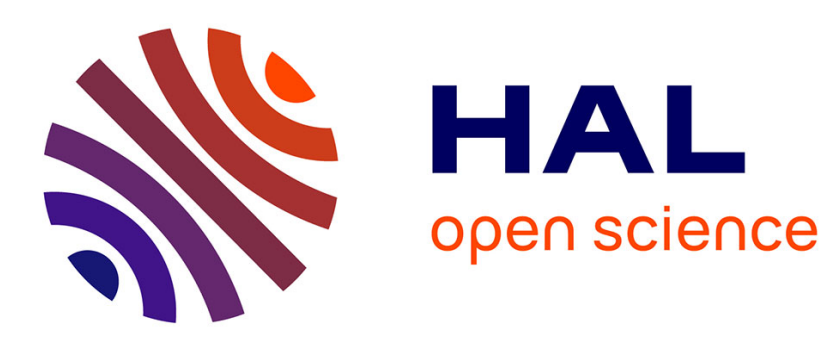

\title{
Simulation of bone tissue formation within a porous scaffold under dynamic compression
}

\author{
Josep A. Planell, Jean-Louis Milan, Josep Planell, Damien Lacroix
}

\section{To cite this version:}

Josep A. Planell, Jean-Louis Milan, Josep Planell, Damien Lacroix. Simulation of bone tissue formation within a porous scaffold under dynamic compression. Biomechanics and Modeling in Mechanobiology, 2010, 9 (5), pp.583 - 596. 10.1007/s10237-010-0199-5 . hal-01635758

\section{HAL Id: hal-01635758 \\ https://hal.science/hal-01635758}

Submitted on 11 Sep 2019

HAL is a multi-disciplinary open access archive for the deposit and dissemination of scientific research documents, whether they are published or not. The documents may come from teaching and research institutions in France or abroad, or from public or private research centers.
L'archive ouverte pluridisciplinaire HAL, est destinée au dépôt et à la diffusion de documents scientifiques de niveau recherche, publiés ou non, émanant des établissements d'enseignement et de recherche français ou étrangers, des laboratoires publics ou privés. 


\title{
Simulation of bone tissue formation within a porous scaffold under dynamic compression
}

\author{
Jean-Louis Milan · Josep A. Planell · Damien Lacroix
}

Received: 1 September 2009 / Accepted: 8 February 2010 / Published online: 4 March 2010

(C) Springer-Verlag 2010

\begin{abstract}
A computational model of mechanoregulation is proposed to predict bone tissue formation stimulated mechanically by overall dynamical compression within a porous polymeric scaffold rendered by micro-CT. Dynamic compressions of $0.5-5 \%$ at $0.0025-0.025 \mathrm{~s}^{-1}$ were simulated. A force-controlled dynamic compression was also performed by imposing a ramp of force from 1 to $70 \mathrm{~N}$. The model predicts homogeneous mature bone tissue formation under strain levels of $0.5-1 \%$ at strain rates of $0.0025-$ $0.005 \mathrm{~s}^{-1}$. Under higher levels of strain and strain rates, the scaffold shows heterogeneous mechanical behaviour which leads to the formation of a heterogeneous tissue with a mixture of mature bone and fibrous tissue. A fibrous tissue layer was also predicted under the force-controlled dynamic compression, although the same force magnitude was found promoting only mature bone during a strain-controlled compression. The model shows that the mechanical stimulation of bone tissue formation within a porous scaffold closely depends on the loading history and on the mechanical behaviour of the scaffold at local and global scales.
\end{abstract}

Keywords Bone tissue engineering - Scaffold .

Tissue differentiation · Mechanoregulation .

Finite element analysis

\section{Introduction}

Biodegradable porous scaffolds open new perspectives in bone tissue engineering and orthopedic surgery to heal

J.-L. Milan (ه) · J. A. Planell · D. Lacroix

Institute for Bioengineering of Catalonia (IBEC),

Technical University of Catalonia,

Barcelona, Spain

e-mail: jean-louis.milan@univmed.fr traumatic bone injuries or replace degenerated osteocartilaginous tissues (Chan and Leong 2008). Porous scaffolds constitute 3D substrates to which the cells can adhere and provide a template for tissue regeneration. Bone tissue engineering requires bioactive scaffolds made of osteoconductive materials or containing growth factors such as bone morphogenetic proteins. The application of mechanical load on cell-seeded scaffolds also stimulates bone tissue formation and may determine the phenotype of the engineered tissue (Ignatius et al. 2005). The definition of an appropriate mechanical environment stimulating bone formation requires, on the one hand, scaffold microstructure optimization to obtain an appropriate mechanical response and, on the other hand, the determination of mechanical loading conditions able to generate within the scaffold the expected mechanical stimuli. These two considerations are necessary to control the mechanical pathway to stimulate bone tissue formation using porous scaffolds.

To regenerate bone tissue, scaffolds can be implanted directly in the body as cell-free structures. In such a case, cells which are recruited from the neighboring tissues to heal the defect site invade scaffold pores, adhere to the walls and synthesize tissue along scaffold geometry (Anderson 2000). After a few days, the scaffold is almost completely colonized by cells, and all the pores are filled by some kind of granulation tissue which will differentiate following biochemical signals but also depending on the mechanical environment (Bolander 1992; Prendergast et al. 1997). In recent years, numerical models of mechanoregulation have analyzed cell differentiation and bone tissue formation within scaffolds (Byrne et al. 2007; McMahon et al. 2008; Sanz-Herrera et al. 2009; Kelly and Prendergast 2006; Liu and Niebur 2008). These studies were based either on the mechanoregulation theories of Carter et al. (1987) and Adachi et al. (2001), or Prendergast et al. (1997) and Lacroix and Prendergast (2002). 
The latter approach relies on the mechanical stimulation of cell differentiation following a criterion based on thresholds of tissue shear strain and interstitial fluid flow velocity (Huiskes et al. 1997). Models of mechanoregulation of cell differentiation were able to predict tissue differentiation in vivo at implant/bone interface (Prendergast et al. 1997), during fracture healing (Lacroix and Prendergast 2002) during mandible distraction osteogenesis (Isaksson et al. 2007), and within bone chamber (Geris et al. 2008; Khayyeri et al. 2009). They could also take into account biological phenomena such as migration and proliferation of cells (Lacroix and Prendergast 2002) or angiogenesis (Checa and Prendergast 2009) as well as scaffold degradation over time (Byrne et al. 2007). Bone ingrowth and tissue phenotype were predicted within idealized or theoretical scaffold geometries submitted to external mechanical loading, and the influence of scaffold material properties such as porosity, stiffness, permeability and dissolution rate was analyzed (Byrne et al. 2007; Kelly and Prendergast 2006; Liu and Niebur 2008). Nonetheless, to the authors' knowledge, tissue formation has not been simulated computationally in real manufactured scaffold designs. In the present study, a model of mechanoregulation of cell differentiation was used to determine the structural influence of manufactured polymeric biodegradable porous scaffolds on tissue phenotype under in vivo conditions of mechanical stimulation. The model was based on the architecture and the material properties of a PLA-Glass scaffold developed by Navarro et al. (2006) and characterized by Charles-Harris et al. (2008) and Charles-Harris et al. (2007). Reconstructed from micro-computed tomography (micro-CT) scans, the porous scaffold was here considered filled by a biological material containing fluid, cells, and matrix and was submitted, via finite element (FE) analysis, to loading conditions reproducing cyclic compression tests performed in vivo within bone chambers (Duty et al. 2007; Case et al. 2003). In this study, the influence of environmental factors other than load for determining cell differentiation is ignored, and it was hypothesized that the formation of tissue with a given phenotype such as mature bone or cartilage within a porous scaffold may be controlled by a given mechanical loading condition.

\section{Methods}

\subsection{FE model of scaffold}

The scaffold was made of polylactic acid (PLA) and titanium-stabilized calcium phosphate glass (Glass) in equal mass quantities and processed by means of the solventcasting salt-leaching method (Navarro et al. 2006). PLAGlass material is a solid foam with high porosity of $95 \%$, maximal pore interconnectivity and large pore diameter in the range of $100-500 \mu \mathrm{m}$. The porous scaffold was shaped as a cylinder of diameter and height of 6 and $7.2 \mathrm{~mm}$, respectively. A finite element model developed in a previous study and based on the scaffold geometry rendered by micro-CT was used in this study (Milan et al. 2009). The volumetric mesh of the rendered scaffold was made of 1.8 million quadratic tetrahedral elements of average size of $73 \mu \mathrm{m}$ (Fig. 1a). The reconstructed scaffold geometry had a high porosity of $90 \%$, and all pores were interconnected. PLA and Glass parts were identified by scaling the gray values of micro-CT and were represented in the model. Relative material properties were assigned to elements of each part (Fig. 1b). The mechanical properties of PLA and Glass were considered linear and isotropic with a Poisson's ratio of 0.33 (Navarro et al. 2006). Because the microporosity of PLA was not taken into account by image rendering, walls of the reconstructed scaffold were represented as dense. As a consequence, Young's moduli of PLA and Glass were adjusted in the model to 0.5 and $10.2 \mathrm{GPa}$, respectively so that the global stiffness of the scaffold was equal to experimental measurement of $130 \mathrm{kPa}$ (Charles-Harris et al. 2007).

\subsection{FE model of biological material}

Biological material containing fluid, cells and matrix was simulated to fill the scaffold pores and was delimited by the walls of a virtual bone chamber which was assumed to be a cylinder of diameter and height of 6.5 and $7.2 \mathrm{~mm}$, respectively (Fig. 1c). A gap between the scaffold and the bone chamber wall which allows in experiments the insertion and removal of the scaffold was reproduced in the model being equal to $0.25 \mathrm{~mm}$. Due to computational limitations and because of global axial symmetry, the volume of the biological material analyzed was reduced to a wedge of $18^{\circ}$ corresponding to $1 / 20$ th of the total volume (Fig. 1d). On the other hand, the scaffold geometry which was highly reticulated was not reduced to preserve internal connections and global unity. The wedge of biological material was meshed into 300,000 elements using MSC Patran software under conditions of congruence with the scaffold mesh. The biological material was considered as a poroelastic material fully saturated with a fluid phase similar to the cell culture Dulbeccos' Modified Eagle Medium at $37^{\circ} \mathrm{C}$ whose density and viscosity are $1 \mathrm{~g} \mathrm{~cm}^{-3}$ and $1.45 \times 10^{-3} \mathrm{~Pa} \mathrm{~s}$, respectively (Bacabac et al. 2005). Elements of the biological material were of quadratic type with three degrees of freedom for displacement and one degree of freedom for pore pressure. The mechanical behavior of the biological material defined in the model followed the poroelastic formulation given by the material library of Abaqus (DS Simulia). The elastic part of change in volume of the porous material was proportional to the logarithm of pressure. Fluid flow velocities through the porous material were given by Darcy's law. The fluid part and solid 
Fig. 1 Reconstruction of a complete scaffold by micro-CT segmentation. a FE scaffold model. b Material property assignment. c Bone chamber walls which enclose the biological material filling scaffold pores. d Final model with a biological material reduced to an axial wedge of $18^{\circ}$

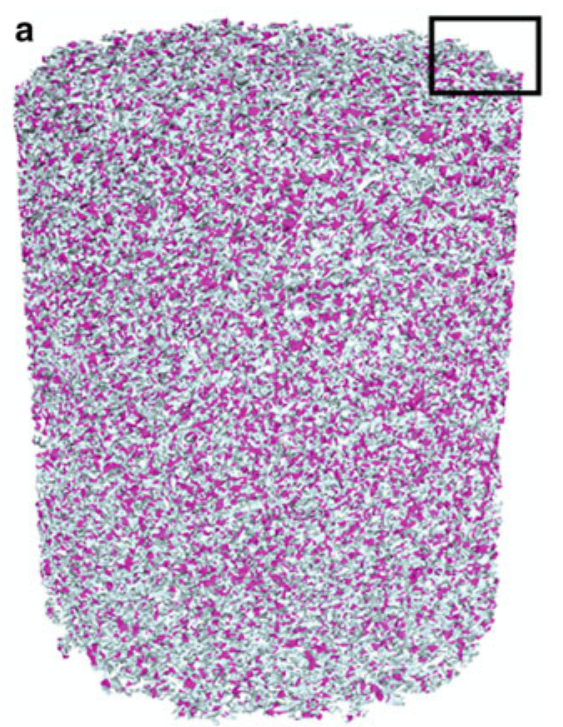

b

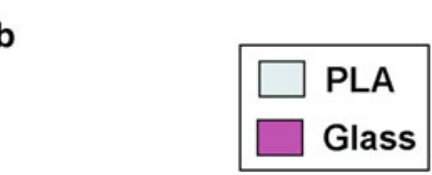

c

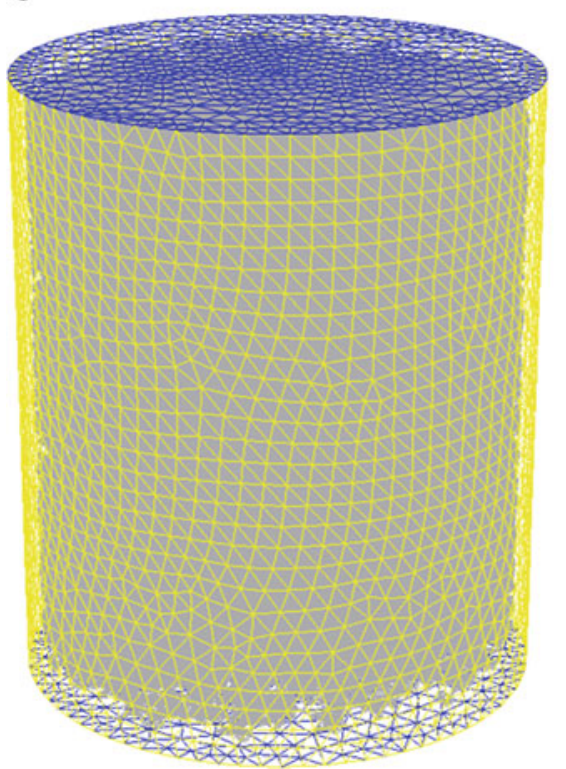

d

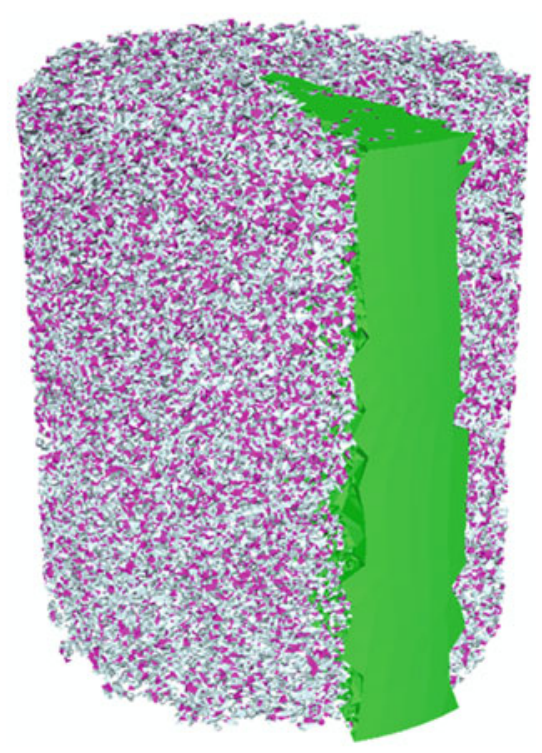

part that composed the tissues were considered in the model as compressible depending on their bulk modulus (Table 1). The bulk modulus of the fluid was set to $2.3 \mathrm{GPa}$ as reported by Anderson (1967), while solid bulk moduli were the ones of the solid part of the different tissues. Tissue permeabilities were taken from experimental measurements in the literature corresponding to tissue macroporosity (Lacroix and Prendergast 2002).

\subsection{FE analysis conditions}

The model of scaffold filled by biological material was submitted to dynamic compression reproducing the major conditions of experimental loading applied in compressive bone chambers such as the one considered by Duty et al. (2007). The cylindrical bone chamber used in vivo confines the scaffold and the forming tissue. Nonetheless, several lateral apertures allow the entrance of fluid and cells from the neighboring tissues into the bone chamber and then into the pores of the scaffold. In the model, the scaffold and the biological material filling the pores were assumed to be confined by the cylindrical wall of the bone chamber by blocking radial displacement of lateral nodes (Fig. 1c, d). The lateral bone chamber wall confined scaffold and tissue but was defined as completely opened for fluid flow as an idealization of the lateral apertures of experimental device. Because of cylindrical symmetry, lateral displacement and fluid flow were constrained on the two inner faces of the biological 
Table 1 Poroelastic material properties of specific tissue phenotypes promoted by the biophysical stimuli, $S$ given by Eq. 1 (Lacroix and Prendergast 2002; Anderson 1967)

\begin{tabular}{llllll}
\hline & Granulation & Mature bone & Immature bone & Cartilage & Fibrous tissue \\
\hline Biophysical stimulus $S$ & $S<0.01067$ & $0.01067<S<0.267$ & $0.267<S<1$ & $1<S<3$ & $3<S$ \\
Young's modulus (MPa) & 0.2 & 6,000 & 1,000 & 0.3 & 0.167 \\
Poisson's ratio & 0.167 & 0.3 & $1 \mathrm{e}-13$ & $5 \mathrm{e}-15$ & 0.167 \\
Permeability $\left(\mathrm{m}^{4} / \mathrm{Ns}\right)$ & $1 \mathrm{e}-14$ & $3.7 \mathrm{e}-13$ & 0.8 & 0.8 & $1 \mathrm{e}-14$ \\
Porosity & 0.8 & 0.8 & 13,920 & 3,400 & 0.8 \\
Solid bulk modulus (MPa) & 2,300 & 13,920 & 2,300 & 2,300 & 2,300 \\
Fluid bulk modulus (MPa) & 2,300 & 2,300 & & 2,300 \\
\hline
\end{tabular}

material wedge. Nil displacement was imposed to bottom nodes. Compression was simulated by imposing force or displacement on top nodes following loading cases described later. FE analysis was computed using Abaqus as a coupled pore fluid diffusion and stress transient analysis using a quasiNewton method.

\subsection{Mechanoregulation algorithm}

Biological material was assumed to be made of stem cells able to differentiate into mature fibroblasts, chondrocytes and osteoblasts. The source of cells did not affect the simulation results. As shown in Fig. 2, the FE model was used in a procedure of mechanoregulation which simulated the process of differentiation of progenitor cells and biological material depending on the mechanical stimuli (Prendergast et al. 1997; Lacroix and Prendergast 2002). The procedure was iterated to reach possible convergence of tissue phenotype under the given mechanical stimulation. The algorithm consisted in calculating, for every iteration, the mechanical stimuli in each element of the biological material, identifying promoted tissue phenotype and updating material properties. For every iteration, FE analysis was performed under the same initial and boundary conditions.

The process of differentiation was regulated by the biophysical stimulus $S$ formulated by Prendergast et al. (1997) and Lacroix and Prendergast (2002) as a combination of tissue shear strain $\varepsilon$ and interstitial fluid flow $v$,

$S=\varepsilon / a+v / b$

in which $a$ and $b$ were equal to $3.75 \%$ and $3 \mu \mathrm{ms}^{-1}$, respectively.

As shown in Fig. 2 and reported in Table 1, four thresholds of $S$ were used to determine whether the precursor cells would differentiate into either fibroblasts, chondrocytes or osteoblasts, leading to the formation of fibrous tissue, cartilaginous tissue or osseous tissue, respectively. If $S<0.01067$, cells would be considered as underloaded, which would lead to tissue resorption and replacement by new granulation tissue.
No predetermined pathways led tissue differentiation from one phenotype to another. The tissue phenotype at iteration $i$ did not depend on the phenotype at iteration $i-1$ but only on $S(i)$. Tissues were assumed to be poroelastic materials whose properties are given by Table 1 .

At the beginning of the simulation, the biological material was entirely constituted of granulation tissue. Ten iterations were performed successively. Cells were assumed to be in confluence within the biological material. Proliferation and migration of cells were not taken into account. Cell apoptosis was simulated by resorbing the existing extracellular matrix into a new granulation tissue. Mechanical properties of the fluid phase within the biological material were assumed to remain unchanged during differentiation simulation.

The model did not take into account any biological time in the simulation of tissue differentiation. Application of mechanical load resulted in a differentiated tissue without considering the duration of stimulation nor the time necessary for cells to synthesize tissue. Thus, the model calculated directly the effect on tissue differentiation of a given mechanical stimulation which must be applied in reality all along the biological process of formation and differentiation. In this study, we analyzed the effect of cyclic compression which is applied over time to scaffolds placed within in vivo bone chamber. Cyclic compression was reproduced in the model as dynamic compression in terms of amplitude and frequency. Differentiation procedure under dynamic compression was repeated to reach algorithm convergence. Procedure iteration was pure algorithmic method and did not reproduce repetition of cycles of real-time compression.

\subsection{Dynamic compressive loading cases}

The model was implemented under dynamic compression considering the same ranges of level and rate of compression as the ones applied cyclically in experimental studies (Duty et al. 2007; Case et al. 2003). Various cases of strain-controlled dynamic compression were simulated. Cases A, B, C were defined, respectively, as $0.5,1$ and $5 \%$ of compression 


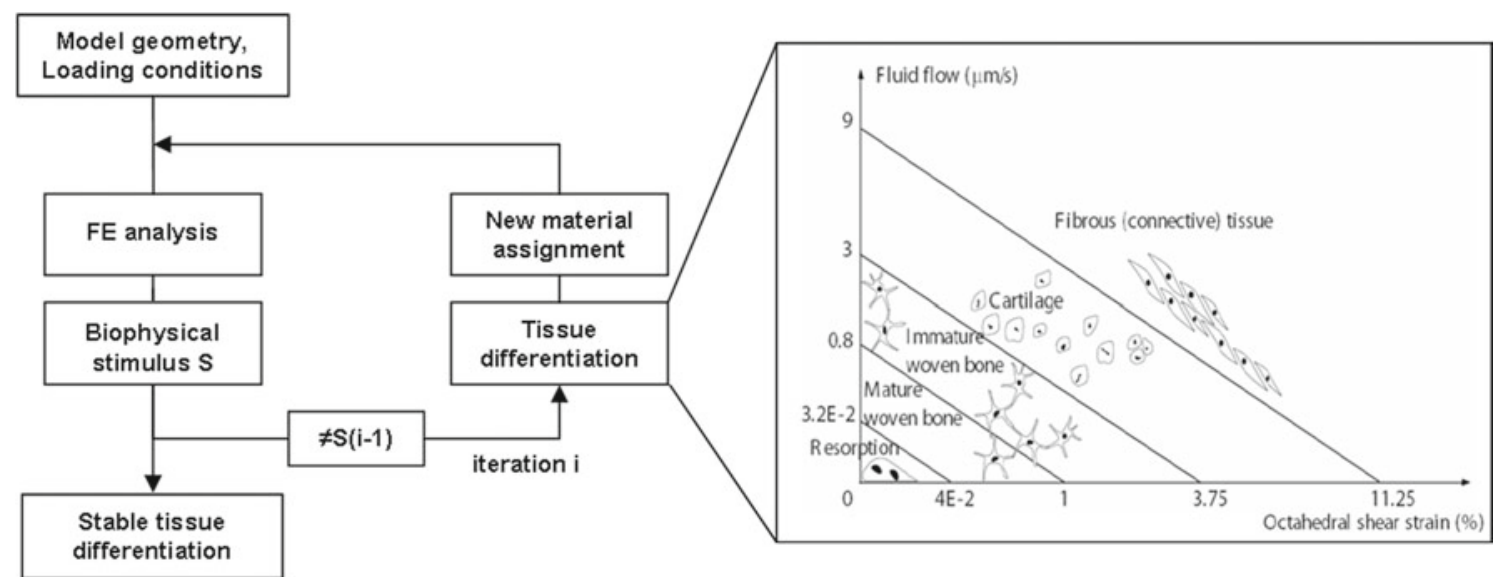

Fig. 2 Algorithm of mechanoregulation. Iterative procedure and diagram of tissue differentiation following thresholds of mechanical stimuli (Prendergast et al. 1997; Lacroix and Prendergast 2002)

by imposing fixed displacements on top nodes during $2 \mathrm{~s}$ leading to strain rates of $0.0025,0.005$ and $0.025 \mathrm{~s}^{-1}$, respectively. Case D consisted in 5\% of compression imposed during $10 \mathrm{~s}$, leading to a strain rate of $0.005 \mathrm{~s}^{-1}$. Case $\mathrm{E}$ consisted in a force-controlled dynamic compression with ramping forces of 1, 2, 5, 10, 20, 30, 40, 50, 60, $70 \mathrm{~N}$ applied subsequently over 10 iterations during $2 \mathrm{~s}$ per iteration. In case $\mathrm{E}$, all the nodes of the top were constrained to the same vertical displacement to avoid rotational movement of the compressive plane. Tissue differentiation within the biological material was expected as a result for the various cases of compression and was analyzed comparing distributions of local strains, stresses and interstitial fluid velocity. A static compression of 5\% was previously performed on the scaffold without biological material inside the pores to analyze the mechanical response of the scaffold alone.

\section{Results}

\subsection{Mechanical response of the FE model}

The mechanical responses of the scaffold with and without biological material inside the pores under $5 \%$ of compression are compared in Fig. 3. In both static and dynamic compressions, scaffold is low strained as shown in Fig. 3a, c. Maximal principal strains are about $0.1 \%$ on average and under $0.5 \%$ except in few pore walls where they can reach a maximum of $12.7 \%$. In the biological material, strains range mainly between $[0.1 ; 60] \%$ and are on average equal to $11 \%$. On the contrary, maximal principal stresses are higher in the scaffold, between $[0 ; 166]$ and $1 \mathrm{MPa}$ on average, than in the biological material, between $[0 ; 80]$ and $17 \mathrm{kPa}$ on average (Fig. 3d). Static compression of 5\% performed on the scaffold alone generates a reaction force of $0.18 \mathrm{~N}$, giving an expected overall stiffness of $130 \mathrm{kPa}$. Under dynamic compression of $5 \%$ performed at a strain rate of $0.025 \mathrm{~s}^{-1}$ on the model composed by the scaffold and the biological material, a reaction force of $0.3 \mathrm{~N}$ was calculated leading to an apparent stiffness of $217 \mathrm{kPa}$. The volume of the scaffold does not deform uniformly under compression. The Fig. $3 \mathrm{~b}$ shows the displacement of the nodes and indicates the local deformation of the volume of the scaffold under $5 \%$ of overall compression. While in some regions the volume deformation does not exceed $-4 \%$, it is about $-20 \%$ at $2 \mathrm{~mm}$ from the base. This means that the scaffold has a heterogeneous compliance, being more compliant in the lower part. The heterogeneous compliance of the scaffold is transmitted to the biological material which is higher strained and stressed in the lower part than in the upper part (Fig. 3c, d). Local strains and stresses range in the upper part between $[0 ; 10] \%$ and $[0 ; 10] \mathrm{kPa}$, respectively, while, in the lower part, they are between $[15 ; 50] \%$ and $[10 ; 166] \mathrm{kPa}$.

The internal distributions of shear strain, fluid velocity and pore pressure within the biological material are shown in Fig. 4. In these distributions, lowest values also are found in the upper part of the model. Shear strain varies mainly between $[0.04 ; 30] \%$, while fluid velocity ranges mainly under $3.2 \times 10^{-5} \mathrm{~mm} \mathrm{~s}^{-1}$. According to the mechanoregulation diagram, formation of immature and mature bone is promoted in the middle of the pores of the scaffold where shear strain and fluid velocity range, respectively, between $[0.04 ; 4] \%$ and $\left[3.2 \times 10^{-7} ; 3 \times 10^{-6}\right] \mathrm{mm} \mathrm{s}^{-1}$, while formation of cartilage and fibrous tissue is promoted close to the pores where shear strain and fluid velocity range respectively between $[15 ; 30] \%$ and $\left[1 \times 10^{-5} ; 1.5 \times 10^{-3}\right] \mathrm{mm} \mathrm{s}^{-1}$. Pore pressure is about $10 \mathrm{kPa}$ in the upper part of the model and reaches $200 \mathrm{kPa}$ in the lower part. Extreme values of pressures and fluid velocities are found near the center of the model. 


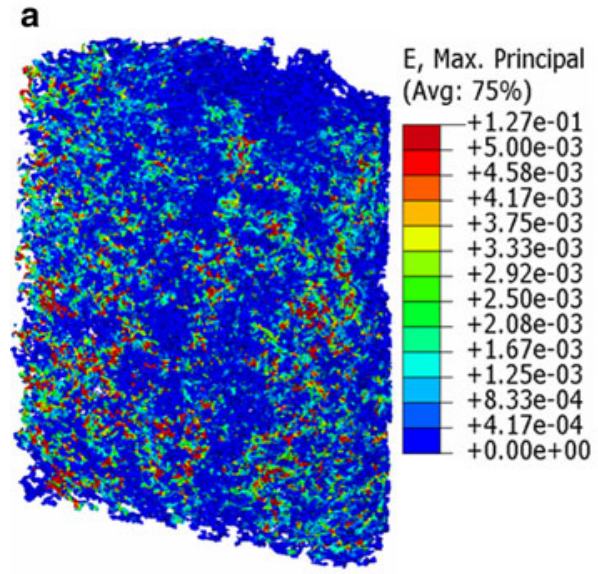

C

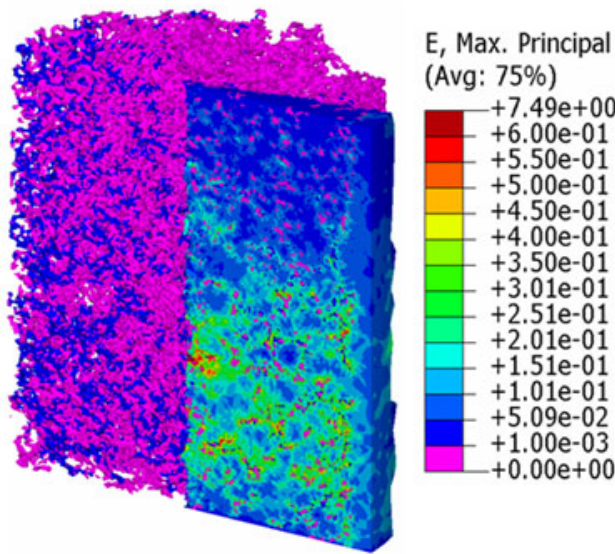

Fig. 3 Deformation of scaffold without (a and $\mathbf{b})$ and with (c and d) biological material inside pores under $5 \%$ of compression. a Internal strains. b Axial displacements $\left(U_{z}\right)$; local deformations of the volume of the scaffold are indicated, being calculated as $\Delta U_{z} / \Delta z$ along a given

\subsection{Tissue differentiation simulation}

Evolution of tissue differentiation over 10 iterations of mechanoregulation algorithm for all cases of loading is shown in Fig. 5. Figures 6 and 7 show, for all cases of loading, the resultant distributions of shear strain and fluid velocity which were computed at the first iteration within the biological material constituted entirely of granulation tissue, and those which were computed through the model updated after 10 iterations. Figure 8 shows the evolution of tissue phenotype proportions within the biological material.

Differentiation of the biological material into mature bone tissue reached $99.5 \%$ in case A and $96 \%$ in case B, corresponding to compression tests of 0.5 and $1 \%$, respectively (Figs. 5a, b, 8a, b). As shown in Fig. 6a, b, at the first iteration, shear strain ranges between $[0.04 ; 3.37] \%$ in $\mathrm{A}$ and between $[0.04 ; 10] \%$ in $\mathrm{B}$, while fluid velocity ranges between $\left[1.75 \times 10^{-7} ; 1 \times 10^{-5}\right] \mathrm{mms}^{-1}$ in

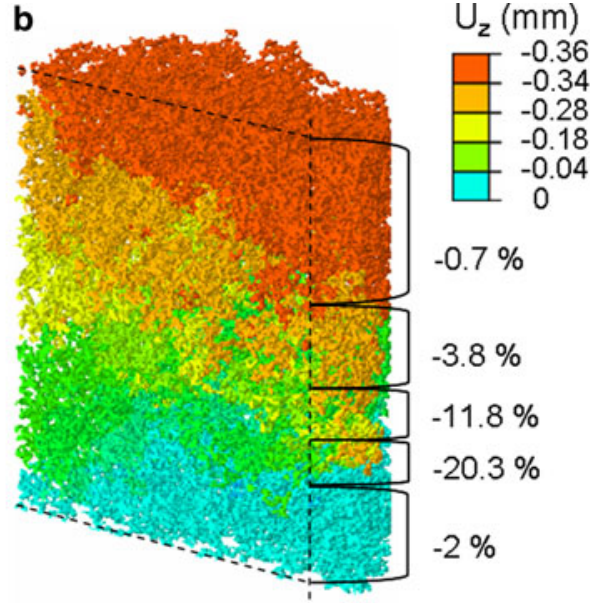

d

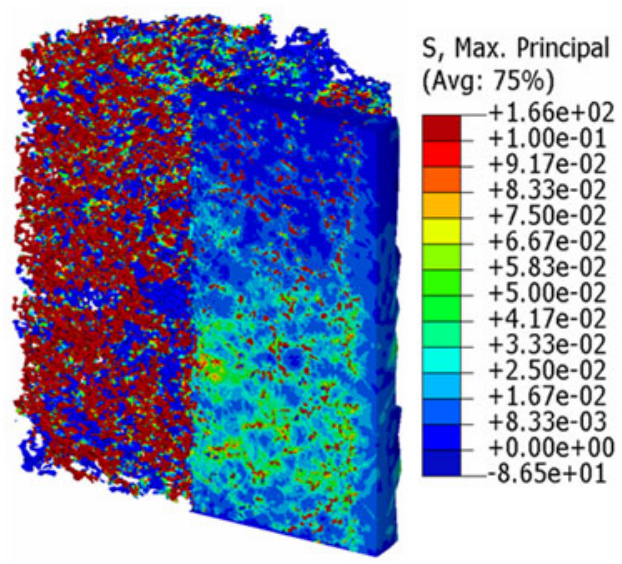

axial direction. $\mathbf{c}$ Maximal principal strains and $\mathbf{d}$ maximal principal stresses within scaffold and biological material under 5\% compression at a strain rate of $0.025 \mathrm{~s}^{-1}$. Cross-sections of the model at the middle of the biological material wedge

A and between $\left[1.75 \times 10^{-7} ; 2 \times 10^{-5}\right] \mathrm{mm} \mathrm{s}^{-1}$ in B. In case $\mathrm{A}$, these ranges of shear strain and fluid velocity give $S$ values belonging to the bone region of the mechanoregulation diagram and lead to a composition of mature bone at $87 \%$ and immature bone at $12.6 \%$ (Fig. 8a). In case $\mathrm{B}$, the differentiated tissue obtained at the first iteration has a higher proportion in immature bone of about $42 \%$, while $1 \%$ of cartilage is formed near scaffold walls (Figs. 6b, 8b).

In case $\mathrm{A}$, the differentiation into mature bone induces an increase in apparent stiffness of the biological material wedge under confined compression, from $6 \mathrm{MPa}$ at the beginning of the simulation to $8.4 \mathrm{GPa}$ at the end, corresponding respectively to reaction force which increases from 0.04 to $70 \mathrm{~N}$. The apparent stiffness of the biological material wedge at the end of simulation in case B is equal to $7.54 \mathrm{GPa}$. Formation of mature bone in almost all the biological material induces an increase in fluid velocity at the end of simulation, especially 
Fig. 4 Distribution of a shear strain, $\mathbf{b}$ fluid flow velocity and c pore pressure within the biological material.

Cross-section of biological material at the middle of the wedge. Axes stand for the center of the scaffold. White void regions indicate unshown scaffold walls

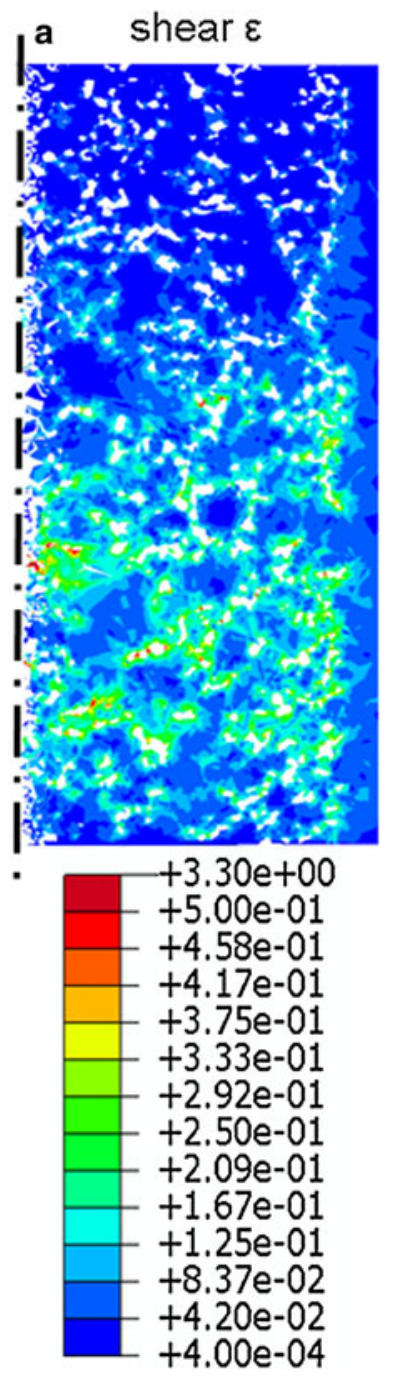

(Strains)

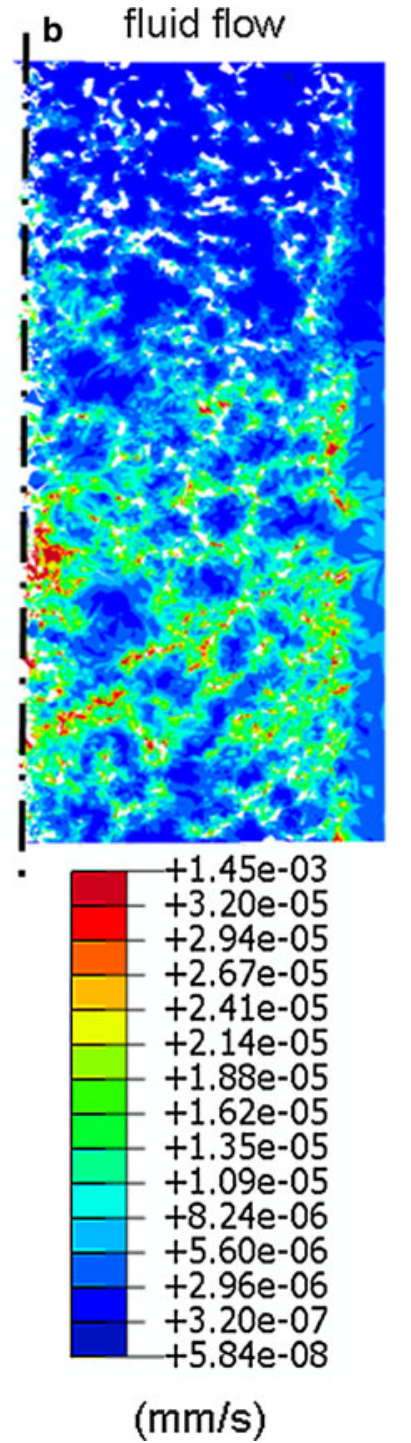

near the outlet zone (Fig. 6a, b). Bone formation in cases A and $B$ induces a homogenization of shear strain in the model (Fig. 6b).

In case $\mathrm{A}$, some local resorption occurs in the mature bone tissue and indicates regions under $0.04 \%$ of strain. These regions show alternately bone formation and bone resorption, indicating that deformation of soft granulation tissue under low stress stimulates formation of bone which, then, in the next iteration, is not strained enough to be maintained. In case $\mathrm{B}$, no resorption was observed within the mature bone, and immature bone is still defined at the equilibrium at the fluid outlet zone.

Applying the same compression level as in case A with a strain rate twice higher led to a distribution of differentiated tissue very closed to the one obtained in case A (results not shown). Applying the case B, in which the level of compression and the strain rate were twice higher than in case A, led to small differences in the distribution of differentiated tissue.
In the range of strain level and strain rate of the following cases: $0.5 \% 0.0025 \mathrm{~s}^{-1}, 0.5 \% 0.005 \mathrm{~s}^{-1}$ and $1 \% 0.005 \mathrm{~s}^{-1}$, the strain level is more determinative than the strain rate for tissue differentiation.

In case $\mathrm{C}$, corresponding to a compression test of $5 \%$ at a strain rate of $0.025 \mathrm{~s}^{-1}$, mature bone and fibrous tissue are the predominant predicted phenotypes (Figs. 5, 8). At the first iteration, due to heterogeneous compliance of scaffold, biological material differentiates into immature bone in the upper part and cartilage and fibrous tissue in the lower part. This amplifies compliance heterogeneity, and, in the next iterations, immature bone and cartilage are replaced by mature bone following a progressive ossification front which is stopped in the lower part by a fibrous tissue layer undertaking almost the entire deformation (Figs. 5, 6). As a result, deformation of the upper part decreases becoming insufficient to maintain mature bone and alternated differentiation is predicted every three iterations changing granulation tissue 


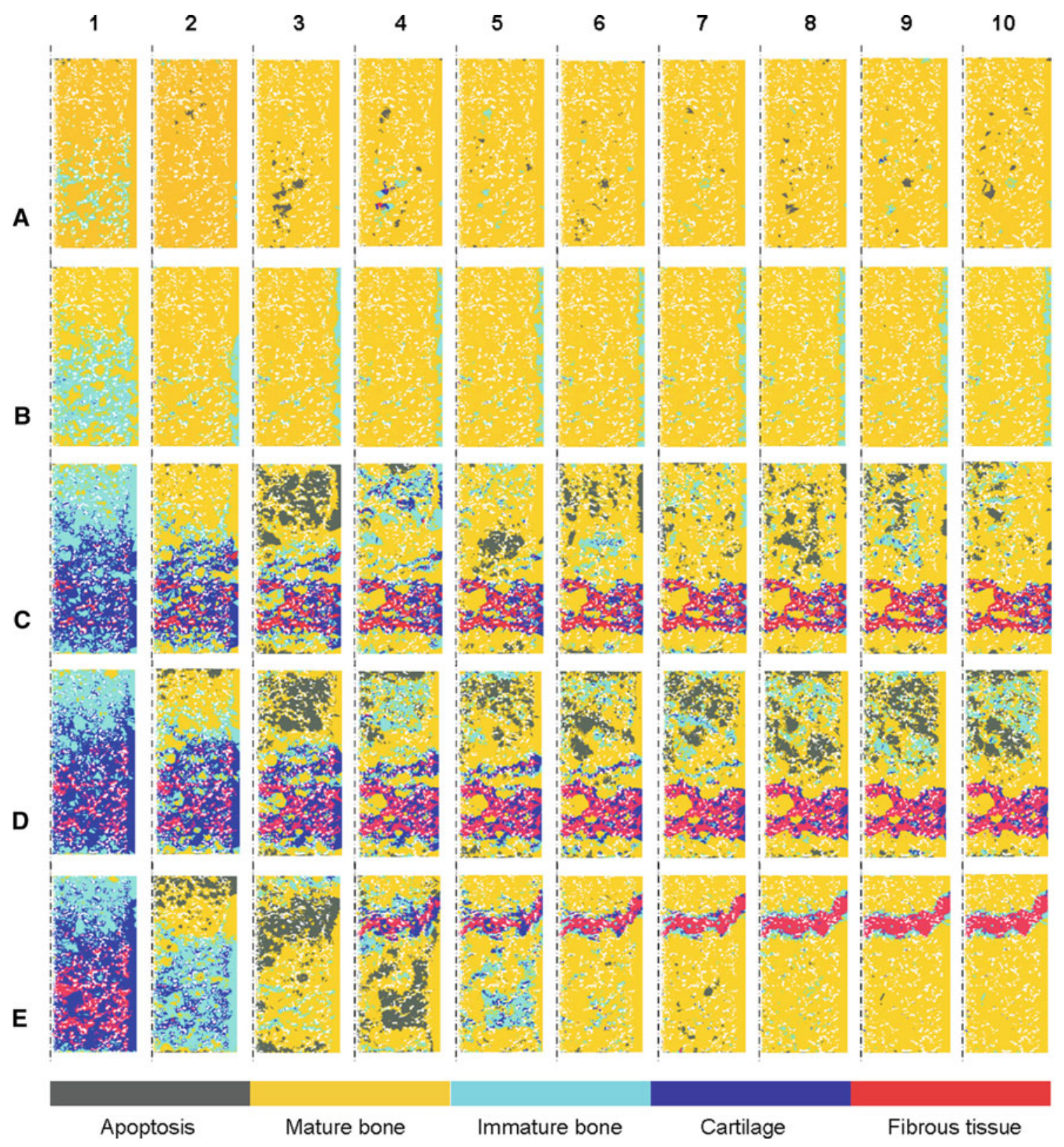

Fig. 5 Tissue differentiation over 10 iterations stimulated by the loading cases: a $0.5 \%$ of compression at a strain rate of $0.0025 \mathrm{~s}^{-1} ; \mathbf{b} 1 \%$ at $0.005 \mathrm{~s}^{-1} ; \mathbf{c} 5 \%$ at $0.025 \mathrm{~s}^{-1} ; \mathbf{d} 5 \%$ at $0.005 \mathrm{~s}^{-1} ; \mathbf{e}$ Ramp of force from

into immature bone, immature bone into mature bone and resorbed mature bone into granulation tissue. This localized alternated differentiation does not interfere with neighboring regions whose phenotype remains unchanged, and after a few iterations, the distribution of tissue phenotypes can be considered as metastable. In case $\mathrm{C}$, due to the presence of fibrous tissue, the apparent stiffness of the biological material wedge at the end of the simulation is only $165 \mathrm{MPa}$ (Fig. 8). Larger amplitudes of compression with higher strain rate $(10 \%$ at $0.05 \mathrm{~s}^{-1}$ and $15 \%$ at $0.075 \mathrm{~s}^{-1}$ ) were tested in the model. Although large deformation formulation may be needed at
1 to $70 \mathrm{~N}$ at rates from 0.5 to $35 \mathrm{~N} \mathrm{~s}^{-1}$. Cross-sections of the biological material at the middle of the wedge; axes stand for scaffold center

such levels of compression, the simulation of differentiation led to more fibrous tissue, and the proportion of fibrous tissue increased with the level of compression (results not shown).

Applying in case $\mathrm{D}$ the same compression level as in case $\mathrm{C}$ at a strain rate five times lower led to small differences in the distributions of shear strain and fluid velocity (Figs. 6c, 7d). As a result, the compositions of differentiated tissues obtained in cases $\mathrm{C}$ and $\mathrm{D}$ at the end of the simulation are similar, and the same fibrous tissue layer is observed in the lower part of the model. Nonetheless, in case D, a larger region of the upper part of the model is submitted to alternated 
Fig. 6 Distributions of shear strain and fluid velocity leading to first differentiation and through the differentiate tissue obtained after 10 iterations for loading cases A, B and E. Distributions are shown in cross-sections of the biological material at undeformed state. Axes stand for scaffold center

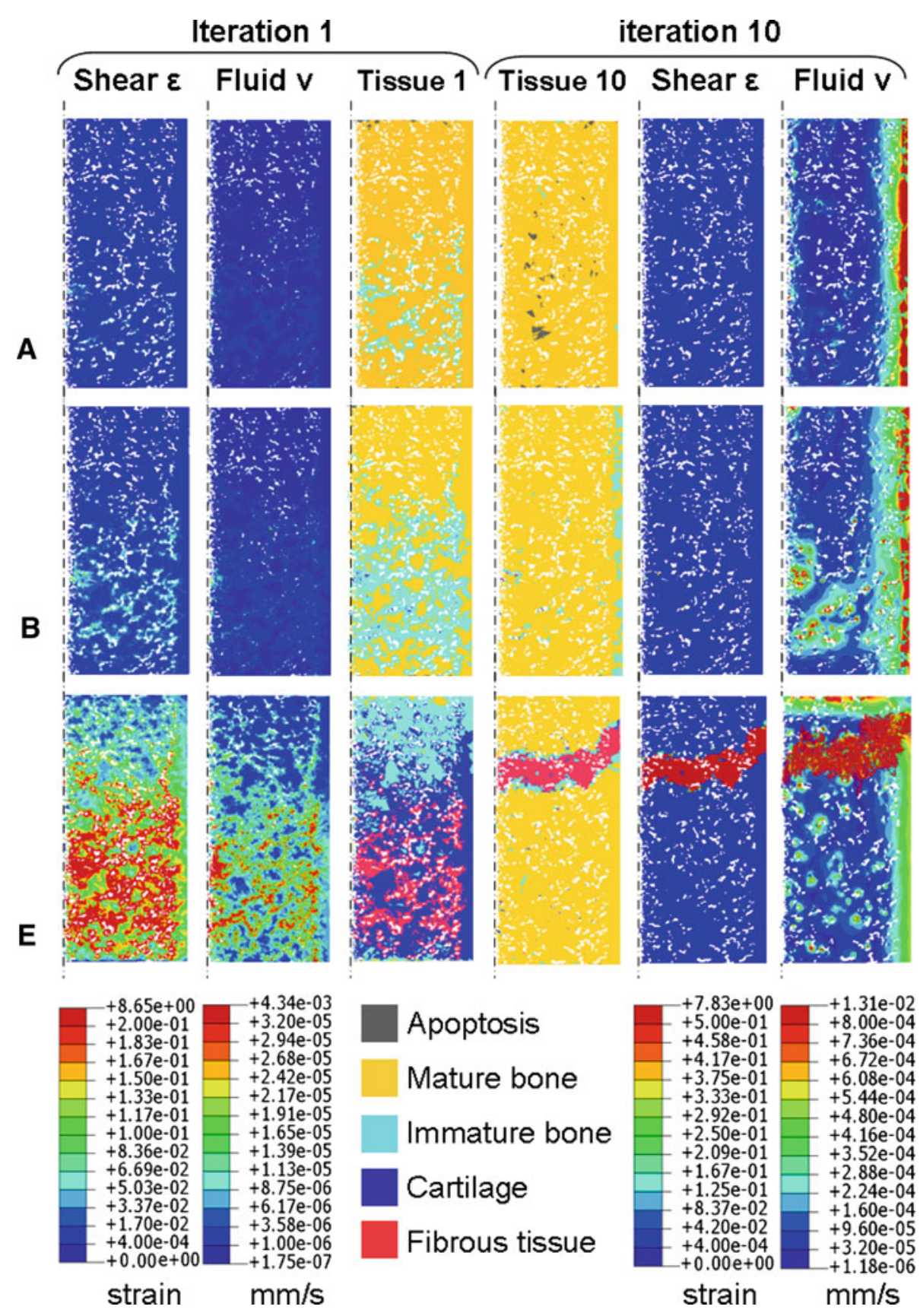

differentiation between formation and resorption of bone, which denotes a lower mechanical stimulation than in case $C$.

The force magnitude of $70 \mathrm{~N}$, which was calculated in case $\mathrm{A}$, was applied in case $\mathrm{E}$ as ramping force. Contrary to case $A$, case $E$ leads to the formation of a fibrous tissue layer in the upper part (Fig. 5e). The application of 1 and $2 \mathrm{~N}$ of force over the first two iterations induces the formation of mature bone in the upper part of the model and immature bone in the lower part. Application of $5 \mathrm{~N}$ in the third iteration leads to mature bone formation in the lower part and resorption of mature bone in a large layer of the upper part, which is replaced by granulation tissue. In iteration 4, this granulation layer, which is highly deformed, differentiates into cartilage and fibrous tissue. Subsequent iterations lead to the formation of only fibrous tissue in that region and mature bone elsewhere. The fibrous tissue layer undergoes the major part of the deformation induced by compressive force, about $15 \%$ under $70 \mathrm{~N}$ at iteration 10 , which leads to an apparent stiffness of the biological material of $260 \mathrm{MPa}$ (Figs. 7e, 8). 
Fig. 7 Distributions of shear strain and fluid velocity leading to first differentiation and through the differentiate tissue obtained after 10 iterations for loading cases $\mathrm{C}$ and $\mathrm{D}$.

Distributions are shown in cross-sections of the biological material at undeformed state. Axes stand for scaffold center

\section{Discussion}

\subsection{Potentialities of model formulation}

One of the main results of this study concerns the stability of the resultant tissue phenotype distribution. Indeed, the regulation of mechanical properties of the model does not follow any relaxing law leading to stable equilibrium but is based on fixed thresholds of mechanical stimulation which were defined independently from the mechanical problem. This demonstrates that the algorithm of mechanoregulation developed by Prendergast et al. (1997) and Lacroix and Prendergast (2002) may lead to an adaptation of the mechanical properties of tissue formed within the scaffold to the overall loading conditions. For instance, the present model predicts the formation of immature or mature bone, cartilage or fibrous tissue depending on the loading conditions. Compressive strains of $0.5-1 \%$ at strain rates of $0.0025-0.005 \mathrm{~s}^{-1}$ induce mature bone differentiation within the pores of the scaffold, while higher strain levels and strain rates of compression lead to mature bone coexisting with fibrous tissue. Because fibrous tissue, cartilage and immature bone possess higher stiffness than the soft granulation tissue, they participate to the decrease of internal strain and help, in that way, the formation of mature bone tissue. This is especially true considering the stabilizing role of the immature bone that leads practically in all the studied cases to mature bone.

Besides, the model shows that bone tissue differentiation within a porous scaffold may depend on the loading history, since the same force level applied via a strain-controlled dynamic compression or a ramping force-controlled dynamic compression does not lead to the same distribution of differentiated tissues.

\subsection{Limitations}

The reduction of the biological material volume due to computational limitations may have induced a stiffer mechanical 
Fig. 8 Proportions of tissue phenotypes over 10 iterations of simulation for all loading cases. Evolution of the apparent stiffness of the biological material wedge
A

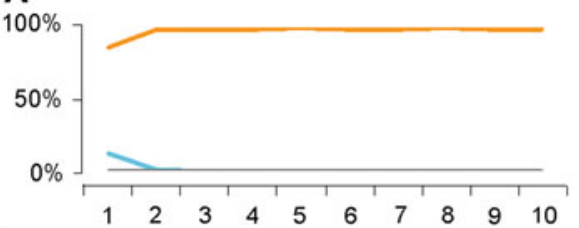

C

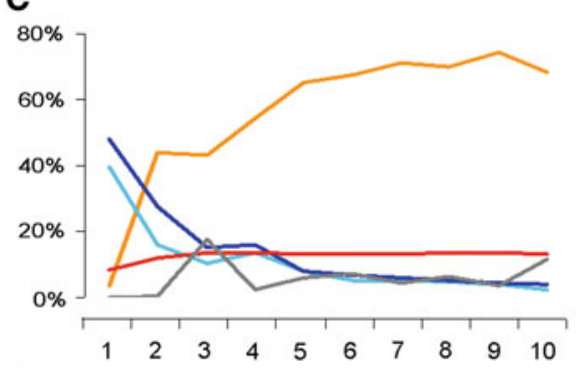

E

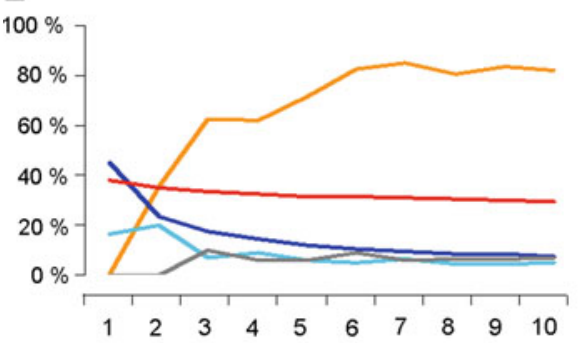

B

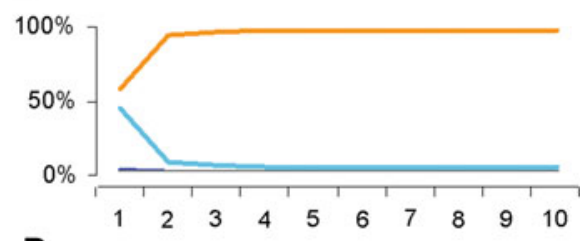

D
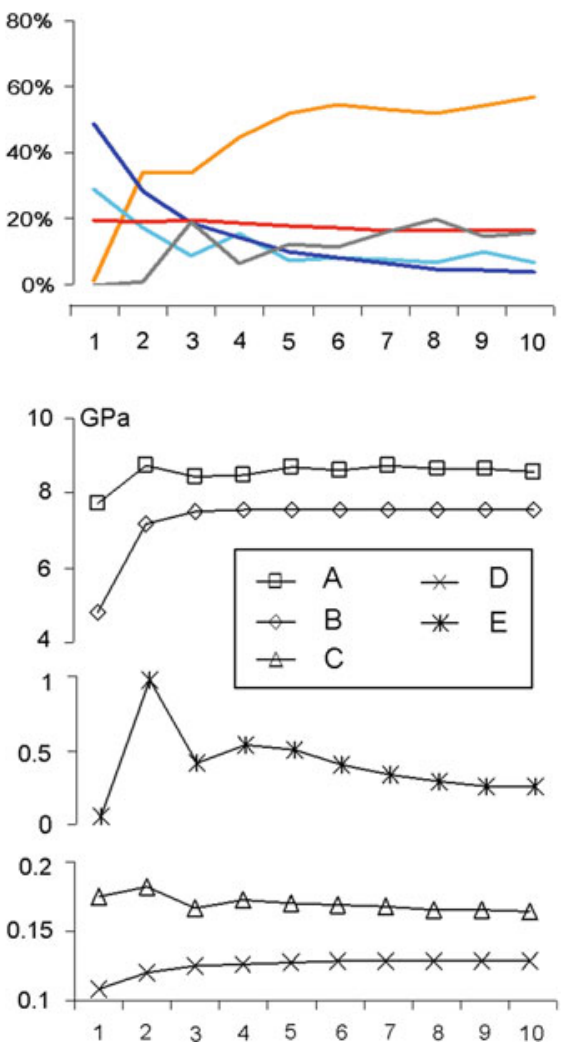

behavior of the scaffold due to the boundary conditions which were applied to block lateral movements of the external nodes of the wedge and which also constrained the scaffold nodes. Although the biological material wedge does not deform uniformly under compression due to a higher compliance of all the lower part of the scaffold, as shown in Fig. 3, this does not depend on the location of the wedge, and the one chosen in the study may be considered as representative.

The use of homogeneous poroelasticity theory to represent bone tissue without taking into account local geometry and microporosity may underestimate the local mechanical environment. Besides, no biological time nor tissue formation rate was considered in the model. Nonetheless, the intermediate results of tissue differentiation during iterative computation may indicate the evolution in time of the differentiated tissue. In fact, one iteration of the mechanoregulation algorithm may correspond to one series of experimental cyclic loading that would last the time necessary for cells to synthesize intermediate tissues.
4.3 Comparison to computational and experimental studies

Results in terms of shear strain and interstitial fluid flow velocity are consistent with those calculated by Lacroix and Prendergast (2002). Fluid velocities are of the same order of magnitude, ranging between $[0.01 ; 10] \mu \mathrm{m} \mathrm{s}^{-1}$. Moreover, the fluid velocities which are involved in mature bone formation are similar between the two studies, ranging between [0.02;0.8] $\mathrm{Mms}^{-1}$. Nonetheless, some discrepancies occur in comparison with the earlier study from our group (Milan et al. 2009) based on the same FE model of scaffold. In that earlier study, the biological material was considered as a fluid, and higher fluid velocities were found under the same level and strain rate of dynamic compression. For instance, $5 \%$ of compression at a strain rate of $0.005 \mathrm{~s}^{-1}$ generated fluid flow velocities in a range of $10-50 \mu \mathrm{ms}^{-1}$ close to scaffold pore walls, which led to fluid flow shear stress about $1 \mathrm{mPa}$ on pore walls. Cartmell (2003) and Porter et al. (2005) identified a relation between cell activity and local fluid shear 
stress in applying perfusion within trabecular bone scaffold. This relation may be used in other structures than trabecular bone and in other loading conditions than perfusion. Following this, the dynamic compression of 5\% at a strain rate of $0.005 \mathrm{~s}^{-1}$ was identified in our previous study as an optimal mechanical loading to stimulate osteo-related response from cells adherent to pore walls. Performing on the present model of mechanoregulation a dynamic compression of 5\% at a strain rate of $0.005 \mathrm{~s}^{-1}$ (case D) leads to the formation of mature bone coexisting with fibrous tissue and partially affected by resorption and cannot constitute here an optimal loading case that stimulates osteogenesis. Instead of considering the biological material as a free-flowing fluid as performed in our earlier study (Milan et al. 2009), the biological material inside scaffold pores was assumed to be composed in this study by a granulation tissue which looks more like a gel and constitutes a preliminary cellular matrix. Fluid flow velocities were calculated using Darcy's law within homogeneous poroelastic elements and are found to be lower than using Navier Stokes equation. Considering that the biological material is likely to evolve from a fluid-like state to gel-like state, parameters used in the algorithm of mechanoregulation may need modification to take into account a greater role of interstitial fluid flow in tissue engineering.

Contrary to the one proposed by Liu and Niebur (2008), the present model of mechanoregulation did not take into account tissue differentiation history or hierarchy among tissue phenotype following imposed pathways from one phenotype to another. Nonetheless, the model showed that in all the various loading cases that were simulated, the tissue differentiation to mature bone may involve succession of the intermediate phenotype from granulation tissue to fibrous tissue and then to immature bone which is observed experimentally. Besides, mature bone did not differentiate in subsequent iterations into another phenotype, unless it was previously resorbed due to underloading. This adaptive bone remodeling is consistent with the mechanostat theory (Frost 1997).

No biological processes such as migration or proliferation of cells, angiogenesis or transport of nutrients or molecules were taken into account in the present model. Tissue differentiation was only determined by mechanical stimuli, and the influence of other local factors such as growth factors or calcium concentration is not taken into account. Obviously, cell proliferation, molecule transport and angiogenesis are challenges of tissue engineering based on scaffolds. Nonetheless, the present model predicts bone formation within the porous scaffold under dynamic compressive strains of $0.5-1 \%$ at strain rates of $0.0025-0.005 \mathrm{~s}^{-1}$, which may be consistent with experimental results. For instance, Bölgen et al. (2008) analyzed the mechanical stimulation of osteoblast-like cells by perfusion and dynamic compression within a scaffold made of lactide and dextran. They showed a positive effect of dynamic compression of $1.5 \%$ at a strain rate of $0.015 \mathrm{~s}^{-1}$ on osteo-related cellular response. Duty et al. (2007) had considered the in vivo mechanical stimulation of osteogenic cells within porous PLA scaffolds implanted subcutaneously in rats. They showed that a dynamic compression of $1.8 \%$ at $0.018 \mathrm{~s}^{-1}$ induced formation and mineralization of bone tissue. Other experimental studies showed that dynamic compression of $5-25 \%$ applied at a strain rate of $0.05-0.25 \mathrm{~s}^{-1}$ to cells cultured in hydrogel scaffolds or ceramic substrates induced synthesis of cartilage matrix proteins (Hung et al. 2004; Kisiday et al. 2004; Waldman et al. 2006). In this study, no cartilage formation was predicted within the scaffold submitted to similar dynamic compressive loadings, and mature bone and fibrous tissue were predicted instead.

\subsection{Tissue distribution within pore space}

In cases $\mathrm{A}$ and $\mathrm{B}$, trabecular mature bone was formed inside scaffold pores. The trabecular mature bone being represented by homogeneous poro-elastic model, the pores of the bone tissue cannot be seen in material distribution. As scaffold and trabecular bone have the same pore size of $100-500 \mu \mathrm{m}$, the only possible physical interpretation is that the solid part of trabecular bone is covering the scaffold pore wall, and the fluid part is located in the middle of pores of the scaffold. This interpretation is consistent with biological observation of tissue deposition within scaffold (Bölgen et al. 2008). Nonetheless, other authors show that bone formation mainly started in the interior of a pore and proceeded toward the scaffold (Vehof et al. 2002). In fact, in the model results, we can see that mechanical stimulus $S$ is highly close to pore walls than that in the middle of the pore. In cases B and C, fibrous tissue and immature bone were found close to pore walls, while mature bone was more located in the middle of the pore. This would mean that after the formation of granulation tissue by the adherent cells from pore wall to the inside, the differentiation of tissue into mature bone may occur first in the middle of the pores, more stable mechanically.

\subsection{Scaffold degradation}

Degradation of polymeric scaffolds over time by biochemical processes is a crucial point in scaffolding for bone tissue engineering but was not taken into account in the present model. Adachi et al. (2006) and Byrne et al. (2007) have simulated bone formation within porous scaffold, taking into account degradability of biomaterial. They showed that scaffold degradation induces mechanical and structural changes in the whole system composed by the scaffold and tissue and may have an influence on the mechanoregulated tissue differentiation. Nonetheless, PLA scaffold shows long degradation time (superior to 12 months) (Rezwan et al. 2006). Degradation 
rates may be reduced in adapting material chemical composition. The composite PLA-Glass scaffold experienced in vitro a weight loss of $25 \%$ after 3 weeks of immersion into simulated body fluid, followed by redeposition of calcium phosphate on scaffold surface (Navarro et al. 2005). After this, no degradation occurs over 6 weeks. Thus, local chemical modifications, such as degradation followed by deposition, may occur in polymeric scaffold during the initial time of immersion, but then degradation may occur after tissue formation and differentiation.

\subsection{Influence of scaffold non-uniformity}

The model derives from the complete geometry of an existing manufactured scaffold which is made of PLA and calcium phosphate glass (Glass) and was previously characterized experimentally (Navarro et al. 2006; Charles-Harris et al. 2008, 2007). Special refinements were involved during scaffold imaging and rendering to represent the whole scaffold structure of a $6 \mathrm{~mm}$ diameter and $7.2 \mathrm{~mm}$ height cylindrical shape and to obtain a valid numerical geometry regarding computational criteria (Milan et al. 2009). Except the neglect of scaffold microporosity which led to a stiffer model, the resultant reconstructed geometry possesses the same fundamental features in terms of macroporosity and pore interconnectivity. Besides, both PLA and Glass phases, which compose the scaffold material, were taken into account and identified in the model. Nonetheless, the model did not take into account the biochemical properties of the PLA-Glass material. Thus, this model is not so specific to PLA-Glass material but more representative of polymeric scaffolds with a porosity of $90-95 \%$. The assumptions of the present model were merely to determine the structural influence of nonuniform polymeric scaffold on the engineered tissue than validate PLA-Glass scaffold.

The present model based on a real scaffold showed heterogeneous compliance of the scaffold. Pores in terms of diameter, densities, interconnectivity and tortuosity were distributed randomly. Heterogeneities in the local mechanical behavior of the scaffold did not show any influence on tissue differentiation, considering low strain levels and strain rates. Nonetheless, at higher strain levels or strain rates, they induced within the scaffold different ranges of mechanical stimulation, which led to heterogeneous distributions of tissue phenotypes. This study shows that under given conditions scaffold heterogeneity can induce heterogeneous mechanical stimuli that lead to heterogeneous tissue formation. This result is particularly relevant for the analysis of tissue engineering experiments in non-uniform scaffolds. This also suggests that sample or patient-specific finite element models are necessary to fully describe experimental results.

\section{Conclusion}

A mechanoregulation model applied to bone tissue engineering using a real scaffold geometry was able to predict formation and differentiation of tissues under loading conditions and could also help in the development of scaffold design. The hypothesis that tissue differentiation could be controlled within a porous scaffold by overall mechanical loading was partially verified. Indeed, mechanical stimulation of bone formation inside a porous scaffold was predicted by the present model; nonetheless, supposedly due to structural heterogeneities, no mechanical loading conditions were identified stimulating cartilage formation. The present study shows that tissue formation and differentiation may be stimulated within a porous scaffold by overall mechanical loading but depends on the mechanical behavior of the scaffold and on the loading history.

Acknowledgments Financial support from the Spanish Ministry of Science and Innovation (MAT2005-07244) is acknowledged.

Conflict of interest statement No competing financial interests exist.

\section{References}

Adachi T, Tsubota KI, Tomita Y, Hollister SJ (2001) Trabecular surface remodelling simulation for cancellous bone using microstructural voxel finite element models. J Biomech Eng 123:403-409

Adachi T, Osako Y, Tanaka M, Hojo M, Hollister SJ (2006) Framework for optimal design of porous scaffold microstructure by computational simulation of bone regeneration. Biomaterials 27(21):39643972

Anderson CB (1967) Mechanics of fluids. In: Baumeister T (ed) Marks' saturated handbook of mechanical engineers, pp 3.48-3.76

Anderson JM (2000) The cellular cascades of wound healing. In: Davies $\mathrm{JE}$ (ed) Bone engineering, Toronto, EM squared, pp 81-93

Bacabac RG, Smit TH, Cowin SC, Van Loon JJWA, Nieuwstadt FTM, Heethaar R, Klein-Nulend J (2005) Dynamic shear stress in parallel-plate flow chambers. J Biomech 38:159-167

Bolander ME (1992) Regulation of fracture repair by growth factors. Proc Soc Exp Biol Med 200:165-170

Bölgen N, Yang Y, Korkusuz P, Güzel E, El Haj AJ, Pişkin E (2008) Three-dimensional ingrowth of bone cells within biodegradable cryogel scaffolds in bioreactors at different regimes. Tissue Eng 14(10):1743-1750

Byrne DP, Lacroix D, Planell JA, Kelly DJ, Prendergast PJ (2007) Simulation of tissue differentiation in a scaffold as a function of porosity, Young's modulus and dissolution rate: application of mechanobiological models in tissue engineering. Biomaterials 28: $5544-5554$

Carter DR, Fyhrie DP, Whalen RT (1987) Trabecular bone density and loading history: regulation of connective tissue biology by mechanical energy. J Biomech 20:785-794

Cartmell SH, Porter BD, Garcia AJ, Guldberg RE (2003) Effects of medium perfusion rate on cell-seeded three-dimensional bone constructs in vitro. Tissue Eng 9(6):1197-1203

Case ND, Duty AO, Ratcliffe A, Müller R, Guldberg RE (2003) Bone formation on tissue-engineered cartilage constructs in vivo: effects of chondrocyte viability and mechanical loading. Tissue Eng 9(4):587-596 
Chan BP, Leong KW (2008) Scaffolding in tissue engineering: general approaches and tissue-specific considerations. Eur Spine J 17(Suppl. 4):467-479

Charles-Harris M, Del Valle S, Hentges E, Bleuet P, Lacroix D, Planell JA (2007) Mechanical and structural characterisation of completely degradable polylactic acid/calcium phosphate glass scaffolds. Biomaterials 28:4429-4438

Charles-Harris M, Koch MA, Navarro M, Lacroix D, Engel E, Planell JA (2008) A PLA/calcium phosphate degradable composite material for bone tissue engineering: an in vitro study. J Mater Sci Mater Med 19:1503-1513

Checa S, Prendergast PJ (2009) A mechanobiological model for tissue differentiation that includes angiogenesis: a lattice-based modeling approach. Ann Biomed Eng 37(1):129-145

Duty AO, Oest ME, Guldberg RE (2007) Cyclic mechanical compression increases mineralization of cell-seeded polymer scaffolds in vivo. J Biomech Eng 129:531-539

Frost HM (1997) Defining osteopenias and osteoporoses: another view (with insights from a new paradigm). Bone 20(5):385-391

Geris L, Vandamme K, Naert I, Vander Sloten J, Duyck J, Van Oosterwyck H (2008) Application of mechanoregulatory models to simulate peri-implant tissue formation in an in vivo bone chamber. J Biomech 41(1):145-154

Huiskes R, Driel WDV, Prendergast PJ, Søballe K (1997) A biomechanical regulatory model for periprosthetic fibrous-tissue differentiation. J Mater Sci Mater Med 8(12):785-788

Hung CT, Mauck RL, Wang CC, Lima EG, Ateshian GA (2004) A paradigm for functional tissue engineering of articular cartilage via applied physiologic deformational loading. Ann Biomed Eng 32(1):35-49

Ignatius A, Blessing H, Liedert A, Schmidt C, Neidlinger-Wilke C, Kaspar D et al (2005) Tissue engineering of bone: effects of mechanical strain on osteoblastic cells in type I collagen matrices. Biomaterials 26(3):311-318

Isaksson H, Comas O, van Donkelaar CC, Mediavilla J, Wilson W, Huiskes R et al (2007) Bone regeneration during distraction osteogenesis: mechano-regulation by shear strain and fluid velocity. J Biomech 40(9):2002-2011

Kelly DJ, Prendergast PJ (2006) Prediction of the optimal mechanical properties for a scaffold used in osteochondral defect repair. Tissue Eng 12(9):2509-2519

Khayyeri H, Checa S, Tägil M, Prendergast PJ (2009) Corroboration of mechanobiological simulations of tissue differentiation in an in vivo bone chamber using a lattice-modeling approach. J Orthop Res 27(12):1659-1666
Kisiday JD, Jin M, DiMicco MA, Kurz B, Grodzinsky AJ (2004) Effects of dynamic compressive loading on chondrocyte biosynthesis in self-assembling peptide scaffolds. J Biomech 37:595-604

Lacroix D, Prendergast PJ (2002) A mechano-regulation model for tissue differentiation during fracture healing: analysis of gap size and loading. J Biomech 35(9):1163-1171

Liu X, Niebur GL (2008) Bone ingrowth into a porous coated implant predicted by a mechano-regulatory tissue differentiation algorithm. Biomech Model Mechanobiol 7(4):335-344

McMahon LA, Reid AJ, Campbell VA, Prendergast PJ (2008) Regulatory effects of mechanical strain on the chondrogenic differentiation of MSCs in a collagen-GAG scaffold: experimental and computational analysis. Ann Biomed Eng 36(2):185-194

Milan JL, Planell JA, Lacroix D (2009) Computational modelling of the mechanical environment of osteogenesis within a polylactic acidcalcium phosphate glass scaffold. Biomaterials 30(25):4219-4226

Navarro M, Ginebra MP, Planell JA, Barrias CC, Barbosa MA (2005) In vitro degradation behavior of a novel bioresorbable composite material based on PLA and a soluble CaP glass. Acta Biomater 1(4):411-419

Navarro M, Aparicio C, Charles-Harris M, Engel E, Ginebra MP, Planell JA (2006) Development of a biodegradable composite scaffold for bone tissue engineering: physicochemical, topographical, mechanical, degradation and biological properties. Adv Polym Sci 200:209-231

Porter B, Zauel R, Stockman H, Guldberg R, Fyhrie D (2005) 3-D computational modeling of media flow through scaffolds in a perfusion bioreactor. J Biomech 38:543-549

Prendergast PJ, Huiskes R, Soballe K (1997) Biophysical stimuli on cells during tissue differentiation at implant interfaces. J Biomech 30:539-548

Rezwan K, Chen QZ, Blaker JJ, Boccaccini AR (2006) Biodegradable and bioactive porous polymer/inorganic composite scaffolds for bone tissue engineering. Biomaterials 27(18):3413-3431

Sanz-Herrera JA, García-Aznar JM, Doblaré M (2009) On scaffold designing for bone regeneration: a computational multiscale approach. Acta Biomater 5:219-229

Vehof JW, Fisher JP, Dean D, van der Waerden JP, Spauwen PH, Mikos AG, Jansen JA (2002) Bone formation in transforming growth factor beta-1-coated porous poly (propylene fumarate) scaffolds. J Biomed Mater Res 60:241-251

Waldman SD, Couto DC, Grynpas MD, Pilliar RM, Kandel RA (2006) A single application of cyclic loading can accelerate matrix deposition and enhance the properties of tissue-engineered cartilage. Osteoarthr Cartilage 14:323-330 